\title{
28 Research Square

\section{Etiologies of delayed diagnosis and characteristics of patients with newly diagnosed advanced lung cancer initially presented with respiratory failure}

\section{Tseng How-Yang}

China Medical University Hospital

\section{Yi-Cheng Shen}

China Medical University Hospital

Yen-Sung Lin

China Medical University Hospital

Chih-Yeh Tu

China Medical University Hospital

Hung-Jen Chen ( $\nabla$ Redman0127@gmail.com )

China Medical University https://orcid.org/0000-0001-6000-9021

\section{Research}

Keywords: Lung cancer, Intensive care unit, Chemotherapy, SOFA, Targeted therapy

Posted Date: April 6th, 2020

DOI: https://doi.org/10.21203/rs.3.rs-20712/v1

License: (c) (1) This work is licensed under a Creative Commons Attribution 4.0 International License. Read Full License 


\section{Abstract}

Objective This study aimed to evaluate the characteristics of patients with newly diagnosed advanced lung cancer who initially presented with respiratory failure. Methods A retrospective study analyzed the outcome of patients in the intensive care unit (ICU) with newly diagnosed advanced lung cancer placed on mechanical ventilation (MV). We defined newly-diagnosed lung cancer as pathological or molecular results for treatment decisions not being out when the patient was admitted to ICU. Results During the 14year inclusion period, 845 lung cancer patients requiring MV were screened. 56 newly diagnosed extensive lung cancer patients were analyzed. Cancer-related to central airway obstruction $(n=29,51.8 \%)$ was the leading cause of respiratory failure. The significant etiologies of delay in the diagnosis of lung cancer were diagnostic error, mistaking the cancer for tuberculosis, and missed hilar lesion. The 6-month survival was only $7.1 \%(n=4)$. SOFA score was significantly associated with mortality $(H R=1.144,95 \% \mathrm{Cl}$ $=1.014-1.291, p=0.028)$. The 6 -month survival rate in patients receiving suitable targeted therapy and accepting chemotherapy and best supportive care was $40 \%(2 / 5), 0 \%(0 / 7)$, and $4.5 \%(2 / 44)$, respectively. Conclusions Patients with newly diagnosed advanced lung cancer with acute life-threatening respiratory failure have poor outcomes. Cancer-related to central airway obstruction is a leading cause of respiratory failure. Diagnostic error as tuberculosis and missed hilar lesions are two main etiologies of delay in the diagnosis. The SOFA score is correlated with mortality. For patients with oncogenic mutation adenocarcinoma, after survival from critical condition, targeted therapy can raise their six month survival rates.

\section{Introduction}

Lung cancer is the most frequent etiology of cancer-related death in recent years [1, 2]. In cancer patients admitted to an intensive care unit (ICU), lung cancer is also the most frequent solid cancer [3]. The primary reason for ICU care is an acute respiratory failure [4-6]. Results for the outcomes of lung cancer patients who need ICU care differ. One study of 49,373 lung cancer patients treated in ICU from Surveillance, Epidemiology, and End Result-Medicare registry showed that ICU outcomes in this study group did not change from 1992 to 2005 [5]. By contrast, outcomes for ICU treatment in lung cancer patients have demonstrated a gradually improving trend due to recent advances in critical care [6, 7]. However, for lung cancer patients who develop acute respiratory failure requiring mechanical ventilation (MV), the mortality rates are around $70 \%[8,9]$.

In recent years, not all types of advanced lung cancer reveal the same poor outcomes. Targeted therapies have changed the prognosis of patients with oncogenic mutated non-small cell lung cancer (NSCLC). Tumors that harbor Epidermal growth factor receptor (EGFR) mutations, chromosomal rearrangements in anaplastic lymphoma kinase (ALK), or c-ros oncogene 1 receptor (ROS1) can present dramatic responses to a targeted tyrosine kinase inhibitor (TKI) [10-14], with less toxicity than with cytotoxic agents. Therefore, for NSCLC patients with an oncogenic mutation in ICU, the best advances in critical care with molecular targeted therapy have raised hopes of a Lazarus treatment response [15-18]. 
For patients with advanced lung cancer within one month of diagnosis, Barth et al. have revealed that ICU management may be helpful, and chemotherapy can improve the prognosis for patients with small cell lung cancer (SCLC) [19]. Chen et al. have also demonstrated that chemotherapy and targeted therapy might afford better ICU survival for treatment-naïve, critically ill lung cancer patients [20].

However, at present no study focuses on critical newly diagnosed lung cancer patients whose pathological or molecular results for treatment decision have not come out when patients admitted to ICU. In this group of ICU patients, many specific considerations deserve concern, including performing interventional procedures (pig-tail catheter drainage, pericardiocentesis or endobronchial electrocautery), selecting of tumor biopsy sites, choosing biopsy methods, determine oncogenic mutation, staging cancer, recovering multiple organ dysfunctions, and giving treatment for lung cancer. Furthermore, we should identify whether there are specific factors that led the patient to develop respiratory failure, even as we remained unable to detect that the patient has lung cancer. With this in mind, we conducted a retrospective study to assess the etiologies of delayed diagnosis and characteristics of patients with newly diagnosed advanced lung cancer who initially presented with respiratory failure.

\section{Materials And Methods}

\section{Study participants}

We performed a retrospective study analyzing the outcomes of patients in the ICU with newly diagnosed advanced lung cancer placed on MV (including noninvasive and invasive ventilation) because of acute life-threatening respiratory failure between January 2006 and January 2019. Advanced lung cancer disease was defined as extensive-stage for SCLC and stage IIIB or IV for NSCLC [21]. Acute lifethreatening respiratory failure was defined as hypoxemia, retention of carbon dioxide, or evidence of respiratory muscle fatigue. Newly-diagnosed lung cancer was defined as final pathology, ALK immunohistochemistry (IHC) or EGFR mutation results for treatment decision not yet out when the patient was admitted to ICU.

Two electronic medical record databases from Tainan Municipal An-Nan Hospital and China Medical University Hospital were included. Patients who were admitted to ICU due to postoperative care or received treatment, i.e., targeted therapy and chemotherapy, before ICU admission were excluded. The Institutional Review Board of China Medical University Hospital approved this study (CMUH 108-REC2136). Informed consent was waived because of the observational and retrospective design.

We classified the main reasons for respiratory failure into direct-lung cancer-related and indirect-lung cancer-induced (e.g., tumor-related central airway obstruction, diffuse pulmonary metastases, brain metastases related increased intracranial pressure, pulmonary embolism, or superior vena cava syndrome) and indirect-lung cancer-related (e.g., pneumonia, cardiac pulmonary edema, severe shock, pneumonia, chronic obstructive pulmonary disease with acute exacerbation, benzodiazepine overdose) events. 


\section{Clinical data collection, Clinical assessments, and Efficacy evaluations}

Demographic and clinical data, including gender, age, comorbidities, smoking history, indication for MV, and do not resuscitate (DNR) orders were collected. Comorbidities were computed with the modified Charlson Comorbidity Index [22]. Patients who had never smoked or who had smoked $<100$ cigarettes in their lifetime were categorized as non-smokers.

Lung cancer-related information, including the histological type, stage, IHC, and EGFR mutation status, were recorded. The pre-ICU (within two weeks before ICU admission) Eastern Cooperative Oncology Group performance status (ECOG-PS) was recorded [23].

The severity of the acute illness was calculated based on data within 24 hours of MV through the Sequential Organ Failure Assessment (SOFA) [24] and Simplified Acute Physiology Score (SAPS) II [25]. The management and treatment for lung cancer patients were classified as best supportive care (BSC), chemotherapy, and targeted therapy (EGFR-TKIs or ALK-TKIs). The adjuvant instrumental procedures to rapidly restore organ functions (e.g., pig-tail catheter drainage for pleural effusions, pericardiocentesis or endobronchial electrocautery) were collected.

Endpoints were 6-month survival rates after the initiation of MV. Furthermore, the administration of anticancer treatments after discharge was described.

\section{Interpretation of chest radiograph}

We also evaluated the series chest radiographs which were taken before the diagnosis of lung cancer to detect the rate of misdiagnosis. Two chest physicians, S.Y.C. and H.J.C., with more than 10 and 20 years of experience in interpretation of chest radiographs, respectively, interpreted all the posterior-anterior chest radiographs for nodular lesions without knowledge of the clinical data. Concordance on the final results was reached after discussions. The delay was defined as the duration between the date of the pathologic diagnosis and the first radiograph on which the missed lesion could be detectable according to the consensus of the two chest physicians.

\section{Statistical analyses}

For clinical data descriptions, continuous variables were presented as medians and interquartile ranges (IQRs) (25th and 75th percentiles), whereas categorical variables were expressed as percentages. Univariate and multivariate analyses for 6-month mortality were performed using the Cox proportional hazards model. Results were expressed as hazard ratios (HRs), with their $95 \%$ confidence intervals ( $95 \%$ $\mathrm{Cls}$ ). The survival time after respiratory failure was estimated using the Kaplan-Meier method, and differences among the subgroups were compared using the log-rank test. A P value of less than 0.05 was set as statistically significant. All statistical analyses were analyzed using the SAS software package (SAS Institute, Inc., Cary, NC). 


\section{Results}

During the 14-year inclusion period, the medical records of 845 lung cancer patients requiring MV were initially screened. Based on the inclusion and exclusion criteria, 56 newly diagnosed extensive lung cancer patients met the criteria and were analyzed (Figure 1). Detailed baseline characteristics are summarized in Table 1. SCLC was diagnosed in $6(10.7 \%)$ patients, squamous cell carcinoma was diagnosed in 16 (28.6\%) patients, and adenocarcinoma was diagnosed in 29 (51.8\%) patient. Out of the 29 adenocarcinoma patients, 10 had active driver mutation, distributed into EGFR mutation $(\mathrm{n}=9)$ and $A L K$ rearrangement $(\mathrm{n}=1)$.

The reasons for respiratory failure are listed in Table 2. Direct-lung cancer-related complications were the main reasons for respiratory failure (43/56,76.8\%), including tumor-related critical airway $(n=29,29 / 56$, $51.8 \%)$, diffuse lung metastases $(n=5)$, brain metastases related increased intracranial pressure $(n=5)$, cardiac tamponade $(n=2)$, pulmonary embolism $(n=1)$ and superior vena cava syndrome $(n=1)$. The other $13(23.2 \%)$ patients were due to indirect-lung cancer-related events. Of these, pneumonia $(n=7)$ was the leading mechanism of respiratory failure.

In 32 of the 56 cases, a previous chest radiograph was available for analysis the etiologies of delayed diagnosis (Figure 2). The median delay was 90 [54.5-191.5] days. Among the 32 patients, one patient refused to accept further exam. In one patient no chest abnormalities could be discovered via the retrospective observation of previous radiologic exams. Of these remaining 30 patients, in $14(46.7 \%)$ abnormalities could be initially detected. However, diagnostic error was made by the attending physician. Of these 14 cases, in 7 (7/14, 50\%), the lesion was misidentified as tuberculosis, in $4(4 / 14,28.6 \%)$ cases lung abscess/pneumonia was the diagnosis, and in $3(3 / 14,21.4 \%)$ cases, these lesions were considered to be cardiogenic pulmonary edema or left atrial enlargement. The others $(n=16,16 / 30,53.3 \%)$ were missed lesions. Eight patients $(8 / 16,50 \%)$ were heavy smokers. Most of the lesions were located in the hilar of the lung $(n=13,13 / 16,81.3 \%)$.

In these 56 newly diagnosed lung cancer patients with respiratory failure, an invasive biopsy was performed during ICU stay for $53.6 \%(n=30)$ of patients. Twenty-two patients $(39.3 \%)$ required adjuvant procedures. Among them, 7 required pleural draining, 4 required pericardiocentesis, and 11 required electrocautery to the tumor-related critical airway. For acute respiratory failure, 45 (80.4\%) patients received invasive MV, and 11 (19.6\%) patients received noninvasive MV (Table 1). Thirty-seven (66.1\%) patients received BSC, 7 (12.5\%) patients received chemotherapy, and $12(21.4 \%)$ received targeted therapy during their ICU stay. No patients with SCLC received chemotherapy.

Of these 12 patients accepting rescue targeted therapy, 5 with oncogenic mutation received suitable TKIs (3 EGFR L858R with erlotinib, 1 EGFR exon 19 deletion with gefitinib and 1 ALK rearrangement with ceritinib), and 1 with EGFR mutation accepted unsuitable EGFR TKI (de novo T790M with erlotinib). The remainder were either wild type $(n=5)$ or had an unknown $(n=1)$ mutation status. However, four patients with EGFR mutation adenocarcinoma did not take targeted therapy, including one patient who refused, while the other three were dead before the molecular report came out. 
The all-cause mortality rate at day 28 after the initiation of MV was $58.9 \%$. For those 23 patients surviving within 28 days, 14 (60.9\%) could not be weaned from the MV. Five patients received chemotherapy therapy, five patients receiving targeted therapy (gefitinib or erlotinib) and 13 patients received BSC. 3-month and 6-month mortality rates were $87.5 \%$ and $92.9 \%$, respectively. Outcomes of the enrolled patients are presented in Figure 1.

Variables found associated with a $p$ value of less than 0.35 in univariate analysis and those considered as clinically relevant (targeted therapy) were entered into a multivariate regression stepwise selection analysis in order to identify the factors independently associated with 6-month mortality. The mortality rates showed no significant differences for smoking, histological type, performance status, indication for MV, adjuvant treatment, DNR orders, and urgent anti-cancer chemotherapy or targeted therapy. Although mortality rates were associated with noninvasive MV in univariate analysis (unadjusted $\mathrm{HR}=3.446, p=$ $0.001)$, this was not significant in the adjusted model $(p=0.598)$. After adjusting for clinical factors, the multivariate analysis showed that the SOFA score was significantly associated with 6-months mortality $(\mathrm{HR}=1.144,95 \% \mathrm{Cl}=1.014-1.291, p=0.028)($ Table 3$)$.

As shown in Table 3, rescue targeted therapy cannot reduce the mortality. However, of these 12 patients accepting rescue targeted therapy, only 5 with oncogenic mutation received suitable TKIs. We further elucidate the survival impact of suitable targeted therapy for patients with oncogenic mutation lung adenocarcinoma. The 6-month survival rate in patients receiving suitable targeted therapy and accepting chemotherapy and BSC was $40 \%(2 / 5), 0 \%(0 / 7)$, and $4.5 \%(2 / 44)$, respectively. Kaplan-Meier analysis (Figure 3 ) also shows a trend toward better survival in patients receiving suitable targeted therapy (logrank test $p=0.059$ ).

\section{Discussion}

The study is the first to focus on the etiologies of delayed diagnosis and characteristics of patients with newly diagnosed advanced lung cancer with acute life-threatening respiratory failure. Cancer-related to central airway obstruction ( $n=29,51.8 \%$ ) was the leading cause of respiratory failure. The significant etiologies of delay in the diagnosis of lung cancer were diagnostic error, mistaking the cancer for tuberculosis, and missed hilar lesion. Twenty-three (41.1\%) patients were surviving at 28 days, and 14 of them $(14 / 23,60.9 \%)$ with ventilator dependence. The 6-month survival was only $7.1 \%(n=4)$ and was associated with the SOFA score. Patients with oncogenic mutation adenocarcinoma may benefit from targeted therapies after they survive critical condition.

Song et al. have demonstrated the number of advanced lung cancer patients admitted to the ICU has increased over time [7]. Zerbib et al. have shown that urgent chemotherapy can benefit patients with lifethreatening complications related to solid neoplasms [26]. For treatment-naïve, critically ill lung cancer patients, chemotherapy and targeted therapy may improve ICU survival $[19,20]$.

However, patients with newly diagnosed advanced lung cancer with acute life-threatening respiratory failure can be considered a distinct group. We defined newly-diagnosed lung cancer as pathological or 
molecular results for treatment decisions not being out when the patient was admitted to ICU. Therefore, patients cannot be given precise treatment immediately. For direct-lung cancer-related respiratory failure, patients may receive invasive diagnostic workup. For indirect-lung cancer-related respiratory failure, ICU physicians must recover the organ dysfunctions related to infection, cardiogenic pulmonary edema, or AECOPD. Unpredicted respiratory failure is another factor associated with poor prognosis in lung cancer patients, especially in those who had no definite diagnosis. Nevertheless, no study has focused on the etiologies of delayed diagnosis and the natural courses of patients with newly diagnosed advanced lung cancer requiring MV support. To address this gap, our research provides informative data.

Our results appear quite different from recent studies on critically ill lung cancer patients $[19,20,26]$ Among these advanced lung cancer patients, the cancer-related critical airway was the leading etiology $(51.8 \%)$ of respiratory failure. Eleven patients need endobronchial electrocautery.

Various factors are associated with mortality for lung cancer patients with respiratory failure $[6,8,27]$. As reported, patients with SCLC have better short-term survival than patients with other types of lung cancer $[19,26]$. In our study, SCLC patients were not associated with mortality because no SCLC patients had good enough activity to receive chemotherapy in ICU. We found that the 6-month mortality rates showed no significant differences for smoking, performance status, indication for MV, adjuvant treatment, DNR orders, and urgent anti-cancer chemotherapy or rescue targeted therapy. However, the acute severity score (SOFA) is associated with the 6-month survival rate (Table 3). This result echoes those of previous reports $[6,8,27]$.

Hsia et al. [27] and Toffart et al. have reported that targeted therapy cannot reduce the early death ( $\leqq 30$ days) mortality [18]. Our study also found that rescue targeted therapy in ICU had no survival benefits (Table 3). This result likely occurred because (1) the severity of the disease is the primary determinant of prognosis in critically ill lung cancer patients, not the cancer itself and (2) of these 12 patients accepting rescue targeted therapy, five without oncogenic mutation and one unknown mutation patient received EGFR-TKIs. Attending physicians adopted the strategy of "shoot first, ask later" $[17,28]$ because they expected that EGFR-TKIs would benefit the status of adenocarcinoma, non-smokers, and East Asian patients [29].

Beyond the critical management with the short-term aim of ICU discharge, patients receiving suitable targeted therapy for oncogenic mutation lung adenocarcinoma have a better survival trend (Fig. 3). Of these five patients, $40 \%$ survived over six months. Our results appear similar to those of a French study led by Toffart. Toffart compared 14 NSCLC treatment naïve patients with oncogenic mutations in ICUs to a non-mutated group and found that the presence of oncogenic mutations is associated with improved late survival [18]. Undoubtedly, targeted therapy in patients requiring MV support and ICU care with targetable oncogenic mutated adenocarcinoma enabled a Lazarus response and prolonged overall survival when patients can overcome the crisis of organ dysfunction.

However, the majority of survivors died within six months ( $n=52,92.9 \%)$. Of these four survivors, two patients were ventilator dependent (Fig. 1). These results raise the question of how to prevent the failure 
to find lung cancer in patients until they develop respiratory failure. In our cohort, the 90-day delayed diagnosis may not have an influence on the stage of lung cancer, but it definitely has an impact on the incidence of respiratory failure. The diagnostic error was $46.7 \%$. Misidentifications as tuberculosis and pneumonia were the leading etiologies. Agrawal et al. have reported that out of 195 patients diagnosed with lung cancer, 79 (40\%) were taking drugs for tuberculosis for at least one month due to the lack of awareness [30]. Lung cancer presenting as lobar or segmental infiltrates has been reported in up to $11 \%$

of patients with delayed resolution of consolidations [31]. A principal concern for physicians in the timing of evaluation of delayed resolution of consolidations may be underlying lung cancer [32]. Missed lesions $(53.3 \%)$ were the other etiology of delayed diagnosis of lung cancer. Overlying anatomic structures, such as hilar, mediastinal structures, ribs, blood vessels, or the heart, are far more critical for missing a nodule in the chest radiograph $[33,34]$. In this study, $81.3 \%$ of missed lesions were located in the hilar of the lung. Cigarette smoking-induced central airway lung cancer overlying to hilar is a crucial factor in missed diagnosis. These can explain why $51.8 \%$ of patients $(n=29)$ had cancer-related central airway obstruction. Cancer-related critical airway can also explain why lung cancer cannot be found in patients until respiratory failure occurs.

Some limitations of this study must be acknowledged. First, the study design was retrospective. Second, twenty-four patients who were transferred from clinics or hospitals had no previous films available for analysis of the etiologies of delayed diagnosis. Third, the sample size was relatively small.

\section{Conclusion}

Based on our results, we find that patients with newly diagnosed advanced lung cancer with acute lifethreatening respiratory failure have poor outcomes. Cancer-related to central airway obstruction is a leading cause of respiratory failure. Diagnostic error as tuberculosis and missed hilar lesions are two main etiologies of delay in the diagnosis. The SOFA score is correlated with mortality. For patients with oncogenic mutation adenocarcinoma, after survival from critical condition, targeted therapy can raise their 6-month survival rates.

\section{Abbreviations}

ALK: anaplastic lymphoma kinase; BSC: best supportive care; DNR: do not resuscitate; ECOG-PS: Eastern Cooperative Oncology Group performance status; EGFR: epidermal growth factor receptor; ICU: intensive care unit; IHC: immunohistochemistry; IQRs: interquartile ranges; NSCLC: non-small cell lung cancer; ROS 1: c-ros oncogene 1; SAPS: Simplified Acute Physiology Score; SCLC: small cell lung cancer; SOFA: Sequential Organ Failure Assessment; TKI: tyrosine kinase

\section{Declarations}

Acknowledgments 
The authors wish to thank Wan-Hua Wu for her technical help in the statistical analysis.

\section{Funding}

Not applicable.

\section{Availability of data and materials}

Not applicable.

\section{Authors' Contributions}

HYT and HJC participated in study conception and design. HYT, YSL, and HJC participated in acquisition of data. YCS and HJC participated in data analysis and interpretation. HYT, YCS, and HJC drafted the manuscript with all authors revising it critically for intellectual content. All authors have read and approved the final version of the manuscript.

\section{Competing interests}

No conflicts exist for the specified authors.

\section{Consent for publication}

All authors have reviewed and approved the manuscript for publication.

\section{Ethics approval and consent to participate}

The Investigational Review Board approved this review at China Medical University Hospital (CMUH 108REC2-136). The requirement of individual patient consent was unnecessary because of the retrospective design.

\section{Author details}

${ }^{1}$ Division of Pulmonary and Critical Care, Department of Internal Medicine, China Medical University Hospital, Taichung, Taiwan. ${ }^{2}$ Department of Internal Medicine, Tainan Municipal An-Nan Hospital.

${ }^{3}$ School of Medicine, College of Medicine, China Medical University, Taichung, Taiwan.

\section{References}

1 Siegel RL, Miller KD, Jemal A. Cancer statistics, 2019. CA: a cancer journal for clinicians 2019; 69:7-34

2 Bray F, Ferlay J, Soerjomataram I, et al. Global cancer statistics 2018: GLOBOCAN estimates of incidence and mortality worldwide for 36 cancers in 185 countries. CA: a cancer journal for clinicians 2018; 68:394-424 
3 Puxty K, McLoone P, Quasim T, et al. Survival in solid cancer patients following intensive care unit admission. Intensive care medicine 2014; 40:1409-1428

4 Adam A, Soubani A. Outcome and prognostic factors of lung cancer patients admitted to the medical intensive care unit. European Respiratory Journal 2008; 31:47-53

5 Slatore CG, Cecere LM, LeTourneau JL, et al. Intensive care unit outcomes among patients with lung cancer in the surveillance, epidemiology, and end results-Medicare registry. Journal of clinical oncology 2012; 30:1686

6 Toffart A-C, Minet C, Raynard B, et al. Use of intensive care in patients with nonresectable lung cancer. Chest 2011; 139:101-108

7 Song JH, Kim S, Lee HW, et al. Effect of intensivist involvement on clinical outcomes in patients with advanced lung cancer admitted to the intensive care unit. PloS one 2019; 14:e0210951

8 Roques S, Parrot A, Lavole A, et al. Six-month prognosis of patients with lung cancer admitted to the intensive care unit. Intensive care medicine 2009; 35:2044

9 Bonomi MR, Smith CB, Mhango G, et al. Outcomes of elderly patients with stage IIIB-IV non-small cell lung cancer admitted to the intensive care unit. Lung Cancer 2012; 77:600-604

10 Mok TS, Wu Y-L, Thongprasert S, et al. Gefitinib or carboplatin-paclitaxel in pulmonary adenocarcinoma. New England Journal of Medicine 2009; 361:947-957

11 Rosell R, Carcereny E, Gervais R, et al. Erlotinib versus standard chemotherapy as first-line treatment for European patients with advanced EGFR mutation-positive non-small-cell lung cancer (EURTAC): a multicentre, open-label, randomised phase 3 trial. The lancet oncology 2012; 13:239-246

12 Kwak EL, Bang Y-J, Camidge DR, et al. Anaplastic lymphoma kinase inhibition in non-small-cell lung cancer. New England Journal of Medicine 2010; 363:1693-1703

13 Shaw AT, Ou S-HI, Bang Y-J, et al. Crizotinib in ROS1-rearranged non-small-cell lung cancer. New England Journal of Medicine 2014; 371:1963-1971

14 Shaw AT, Kim D-W, Mehra R, et al. Ceritinib in ALK-rearranged non-small-cell lung cancer. New England Journal of Medicine 2014; 370:1189-1197

15 Langer CJ. The "Lazarus response" in treatment-naive, poor performance status patients with nonsmall-cell lung cancer and epidermal growth factor receptor mutation. J Clin Oncol 2009; 27:1350-1354

16 Chien $\mathrm{C}-\mathrm{R}$, Chen $\mathrm{H}-\mathrm{J}$. Lazarus response to treatment of patients with lung cancer and oncogenic mutations in the intensive care unit. Journal of thoracic disease 2016; 8:E1455 
17 Kerrigan K, Shoben A, Otterson G. Treatment of lung cancer patients with actionable mutations in the intensive care unit. Clinical lung cancer 2016; 17:523-527

18 Toffart A-C, Dhalluin X, Girard N, et al. Patients with advanced lung cancer harboring oncogenic mutations should be admitted to intensive care units. Intensive care medicine 2015; 41:164-165

19 Barth C, Soares M, Toffart A, et al. Characteristics and outcome of patients with newly diagnosed advanced or metastatic lung cancer admitted to intensive care units (ICUs). Annals of intensive care $2018 ; 8: 80$

20 Chen Y-F, Lin J-W, Ho C-C, et al. Outcomes of cancer therapy administered to treatment-naïve lung cancer patients in the intensive care unit. Journal of Cancer 2017; 8:1995

21 Goldstraw P, Crowley J, Chansky K, et al. The IASLC Lung Cancer Staging Project: proposals for the revision of the TNM stage groupings in the forthcoming (seventh) edition of the TNM Classification of malignant tumours. Journal of thoracic oncology $2007 ; 2: 706-714$

22 Charlson ME, Pompei P, Ales KL, et al. A new method of classifying prognostic comorbidity in longitudinal studies: development and validation. Journal of chronic diseases 1987; 40:373-383

23 Oken MM, Creech RH, Tormey DC, et al. Toxicity and response criteria of the Eastern Cooperative Oncology Group. American journal of clinical oncology 1982; 5:649-656

24 Vincent J-L, Moreno R, Takala J, et al. The SOFA (Sepsis-related Organ Failure Assessment) score to describe organ dysfunction/failure. Intensive care medicine 1996; 22:707-710

25 Le Gall J-R, Lemeshow S, Saulnier F. A new simplified acute physiology score (SAPS II) based on a European/North American multicenter study. Jama 1993; 270:2957-2963

26 Zerbib Y, Rabbat A, Fartoukh M, et al. Urgent chemotherapy for life-threatening complications related to solid neoplasms. Critical care medicine 2017; 45:e640-e648

27 Hsia T-C, Tu C-Y, Chen H-J. The impact of rescue or maintenance therapy with EGFR TKIs for Stage IIIb-IV non-squamous non-small-cell lung cancer patients requiring mechanical ventilation. BMC anesthesiology $2014 ; 14: 55$

28 Bosch-Barrera J, Sais E, Lorencio C, et al. Successful empirical erlotinib treatment of a mechanically ventilated patient newly diagnosed with metastatic lung adenocarcinoma. Lung Cancer 2014; 86:102-104

29 Thatcher N, Chang A, Parikh P, et al. Gefitinib plus best supportive care in previously treated patients with refractory advanced non-small-cell lung cancer: results from a randomised, placebocontrolled, multicentre study (Iressa Survival Evaluation in Lung Cancer). The Lancet 2005; 366:15271537 
30 Agrawal A, Agarwal P, Tandon R, et al. Pulmonary tuberculosis as a confounder for bronchogenic carcinoma due to delayed and misdiagnosis. Indian Journal of Community Health 2013; 25:438-444

31 Feinsilver SH, Fein AM, Niederman MS, et al. Utility of fiberoptic bronchoscopy in nonresolving pneumonia. Chest 1990; 98:1322-1326

32 Johnson JL. Slowly resolving and nonresolving pneumonia: questions to ask when response is delayed. Postgraduate medicine 2000; 108:115-122

33 Quekel LG, Kessels AG, Goei R, et al. Miss rate of lung cancer on the chest radiograph in clinical practice. Chest 1999; 115:720-724

34 Muhm JR, Miller W, Fontana R, et al. Lung cancer detected during a screening program using fourmonth chest radiographs. Radiology 1983; 148:609-615

\section{Tables}

Table 1. Patients' characteristics 


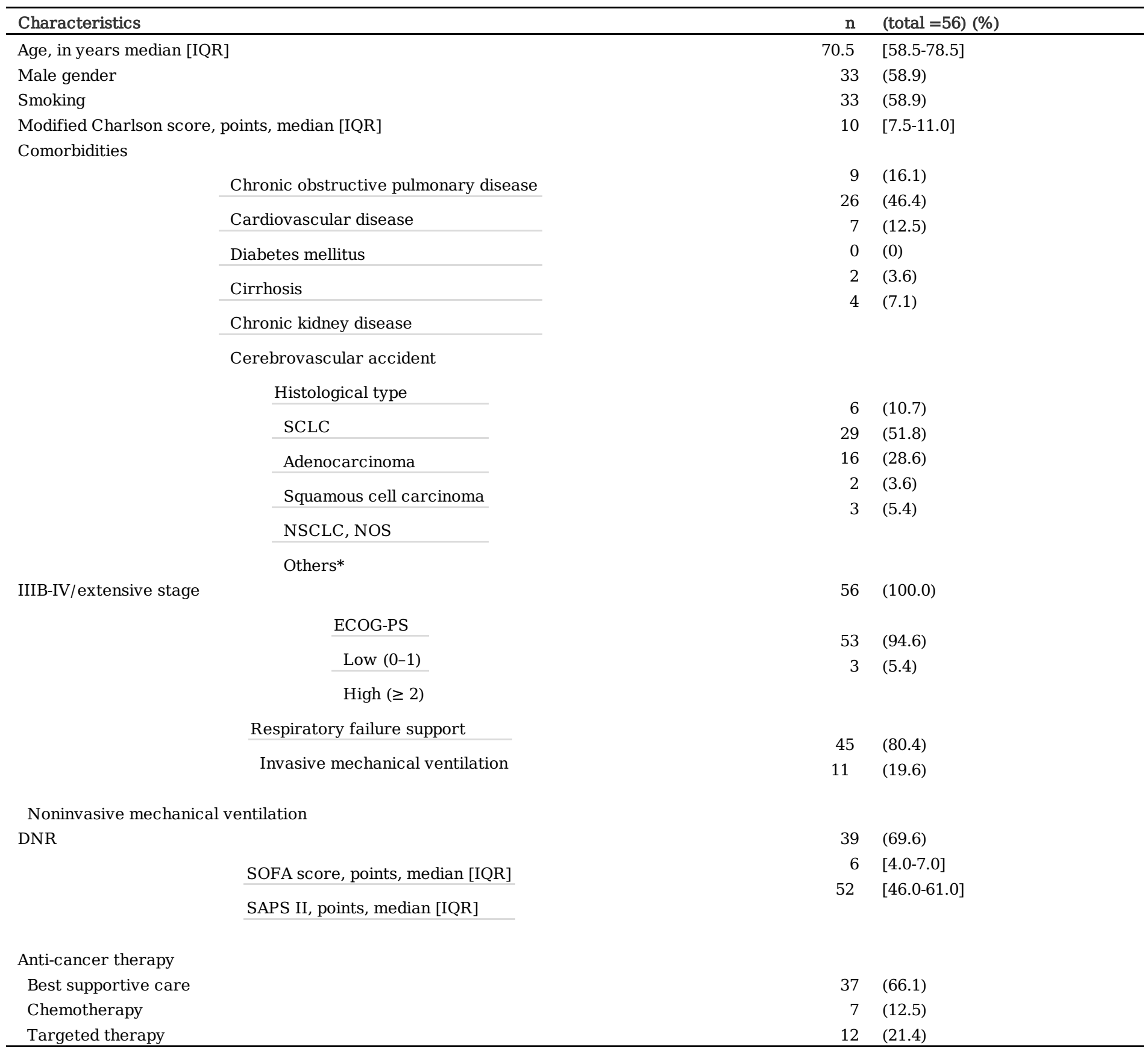

DNR, Do not resuscitation; ECOG-PS, Eastern Cooperative Oncology Group performance status; IQR, interquartile range; NOS, not otherwise specified; NSCLC, non-small cell lung cancer; SAPS II, Simplified Acute Physiology Score, version II; SCLC, small cell lung cancer; SOFA, Sequential Organ Failure Assessment

* One patient has large cell carcinoma, one patient has sarcomatoid carcinoma, and one patient has adenosquamous carcinoma.

Table 2. Major indication for mechanical ventilator 


\begin{tabular}{|c|c|c|}
\hline Characteristics & $\mathrm{n}($ total $=56)$ & $(\%)$ \\
\hline Direct lung cancer related events & 43 & \multirow[t]{6}{*}{$(76.8)$} \\
\hline Central airway obstruction & $\begin{array}{r}29 \\
5\end{array}$ & \\
\hline Diffuse lung metastasis & 5 & \\
\hline Brain metastasis & 2 & \\
\hline Cardiac tamponade & \multirow{2}{*}{$\begin{array}{l}1 \\
1\end{array}$} & \\
\hline Superior vena cava syndrome & & \\
\hline \multicolumn{3}{|l|}{ Pulmonary embolism } \\
\hline Indirect lung cancer related events & $\begin{array}{r}13 \\
7\end{array}$ & $(23.2)$ \\
\hline Pneumonia & \multicolumn{2}{|l|}{3} \\
\hline Pulmonary edema & \multirow{4}{*}{$\begin{array}{l}1 \\
1 \\
1\end{array}$} & \\
\hline Septic shock & & \\
\hline COPD with acute exacerbation & & \\
\hline Drug overdose & & \\
\hline
\end{tabular}

Table 3. Multivariate analysis of clinical factors associated with 6-month mortality

CI, confidence interval, DNR, Do not resuscitation; ECOG-PS, Eastern

Cooperative Oncology Group performance status; IQR, interquartile range; NSCLC, non-small cell lung cancer; Ref, reference; SAPS II, Simplified Acute Physiology Score, version II; SCLC, small cell lung cancer; SOFA, Sequential Organ Failure Assessment

\section{Figures}




\begin{tabular}{|c|c|c|c|c|c|c|}
\hline \multirow[t]{2}{*}{ Clinical factors } & \multirow{2}{*}{$\begin{array}{c}\text { No. of } \\
\text { patients }\end{array}$} & \multicolumn{2}{|c|}{ Univariable analysis } & \multicolumn{3}{|c|}{ Multivariable analysis } \\
\hline & & Hazard Ratio & $p$-value & Hazard Ratio & $95 \% \mathrm{CI}$ & $p$-value \\
\hline \multicolumn{7}{|l|}{ Smoking } \\
\hline No & 23 & Ref & & Ref & & \\
\hline Yes & 33 & 1.337 & 0.308 & 1.522 & $0.778-2.975$ & 0.220 \\
\hline \multicolumn{7}{|l|}{ Histological type } \\
\hline SCLC & 39 & $\begin{array}{r}\mathrm{KeI} \\
1.229\end{array}$ & 0.668 & & & \\
\hline No/unknown drive mutation NSCLC & 10 & 0.817 & 0.724 & & & \\
\hline Driver mutation NSCLC & 01 & 1.120 & 0.919 & & & \\
\hline \multicolumn{7}{|l|}{ Others } \\
\hline ECOG-PS & 52 & & & $\mathrm{P}$ & & \\
\hline Low $(0-1)$ & 03 & 0.374 & 0.175 & 0.584 & $0.136-2.508$ & 0.470 \\
\hline $\operatorname{High}(\geq 2)$ & & & & & & \\
\hline \multicolumn{7}{|l|}{ Severity score } \\
\hline SOFA score, points, median [IQR] & & $\begin{array}{l}1.096 \\
1.006\end{array}$ & $\begin{array}{l}0.099 \\
0.681\end{array}$ & 1.144 & 1.014-1.291 & 0.028 \\
\hline \multicolumn{7}{|l|}{ SAPS II, points, median [IQR] } \\
\hline \multicolumn{7}{|l|}{ Indication for mechanical ventilation } \\
\hline Indirect cancer related & 43 & 0.830 & 0.574 & & & \\
\hline \multicolumn{7}{|l|}{ Direct cancer related } \\
\hline \multicolumn{7}{|l|}{ Respiratory failure support } \\
\hline Invasive mechanical ventilation & $\begin{array}{l}45 \\
11\end{array}$ & $\begin{array}{r}\text { KeI } \\
3.446\end{array}$ & 0.001 & 1.796 & $0.203-15.866$ & 0.598 \\
\hline \multicolumn{7}{|l|}{ Noninvasive mechanical ventilation } \\
\hline Pig-tail catheter/Pericardiocentesis & 45 & Ref & & Ref & & \\
\hline $\begin{array}{l}\text { No } \\
\text { Yes }\end{array}$ & 11 & 0.706 & 0.344 & 0.864 & 0.375-1.992 & 0.732 \\
\hline \multicolumn{7}{|l|}{ Electrocautery } \\
\hline No & 45 & Ref & & & & \\
\hline Yes & 11 & 0.799 & 0.527 & & & \\
\hline \multicolumn{7}{|l|}{ DNR } \\
\hline No & 17 & Ref & & & & \\
\hline Yes & 39 & 1.068 & 0.830 & & & \\
\hline \multicolumn{7}{|l|}{ Chemotherapy } \\
\hline No & 49 & Ref & & & & \\
\hline Yes & 07 & 0.729 & 0.439 & & & \\
\hline \multicolumn{7}{|l|}{ Targeted therapy } \\
\hline No & 44 & Ref & & Ref & & \\
\hline Yes & 12 & 0.827 & 0.591 & 0.623 & 0.250-1.556 & 0.311 \\
\hline
\end{tabular}




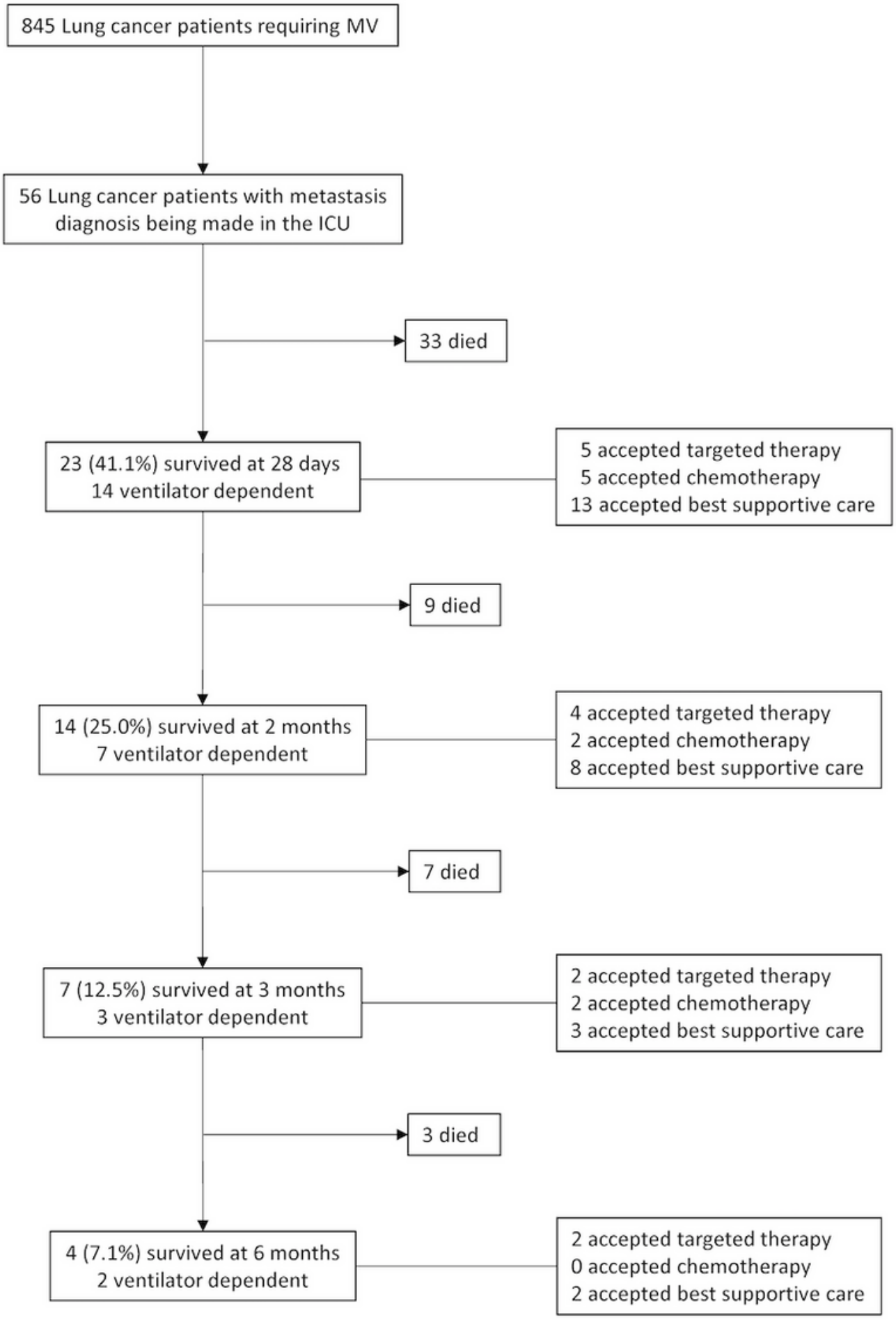

\section{Figure 1}

Flowchart of enrolled subjects. MV, mechanical ventilation; ICU, intensive care unit; COPD, chronic obstructive pulmonary disease 


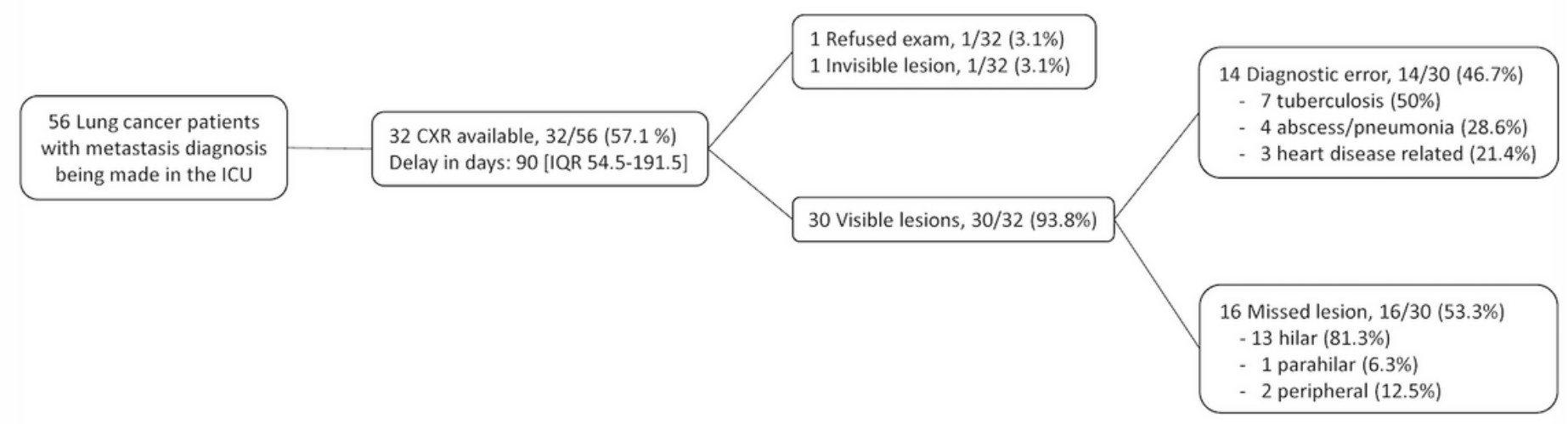

\section{Figure 2}

Flowchart of a previous chest radiograph is available for analysis the etiologies of delay in the diagnosis of lung cancer. CXR, chest X-ray; ICU, intensive care unit; IQR, interquartile range

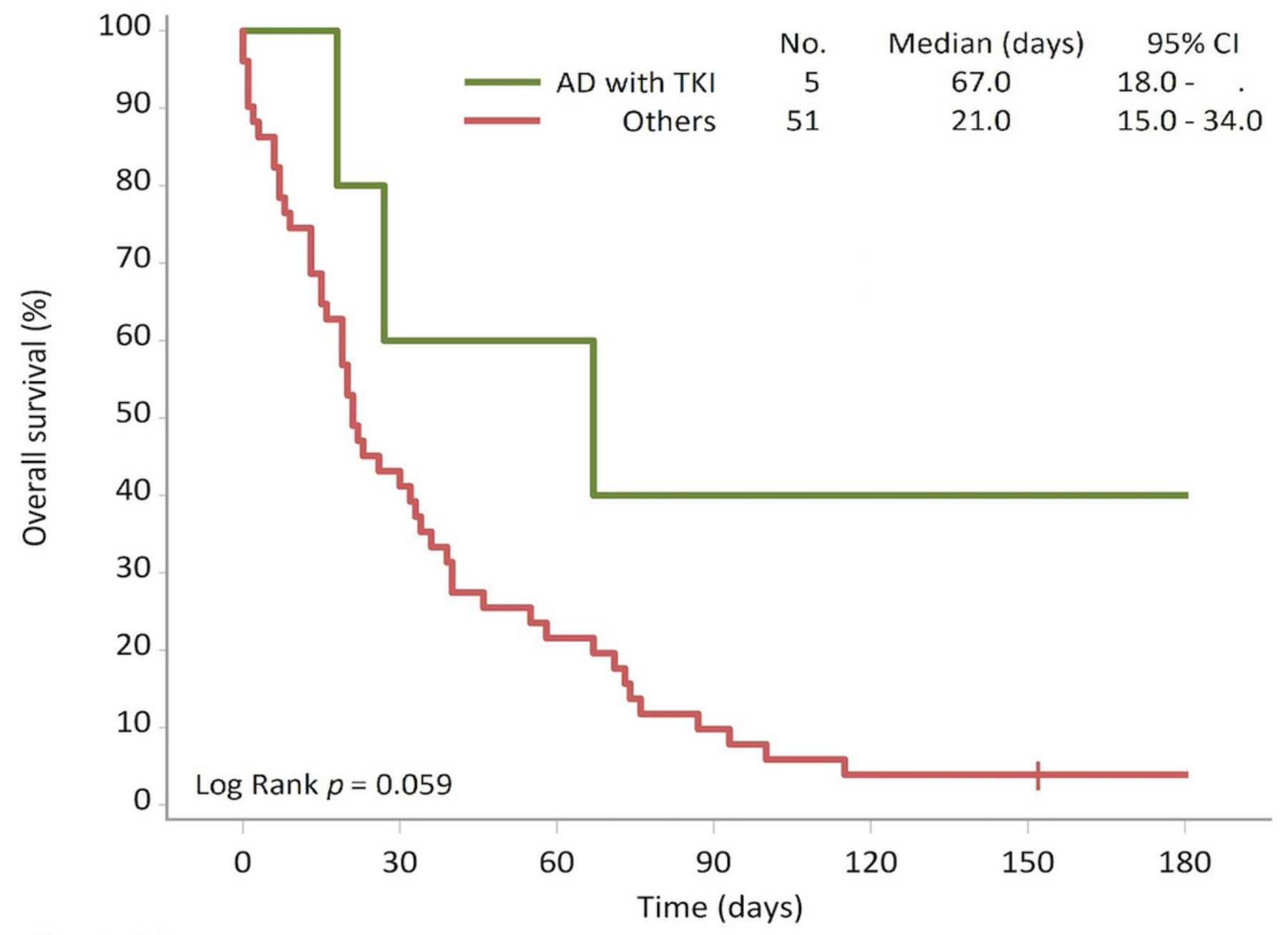

No. at risk

53

3

2

2

2

2

Others

51

22

11

5

2

2

1 


\section{Figure 3}

Kaplan-Meier survival curve according to the cancer treatment. AD with TKI, patients receiving suitable targeted therapy for oncogenic mutation lung adenocarcinoma; Others, the other lung cancer patients 